\title{
Influencia del modelo pedagógico por alternancia en el desarrollo local de cinco comunidades rurales del departamento de Petén, Guatemala
}

\section{Tiklay yaćhana mudilukap kuyuchinin kiki kaynin wiñachinin picha ćhakla ayllukunap Petén suyupiita, Guatemala}

\section{Okantagani ora jigoagantsi kara 5 okibianë timagantsipage kara atirijegikë Peten- Guatemara}

Recepción: 29 septiembre 2020 Corregido: 16 diciembre 2020 Aprobación: 27 febrero 2020

Deysi Lisbeth Rodriguez Max

Nacionalidad: guatemalteca / Universidad de San Carlos de Guatemala

Centro Universitario de Petén. / Correo: 20130909@profesor.usac.edu.gt

Orcid: https://orcid.org/oooo-0003-0965-090X

\section{Resumen}

Este artículo presenta los resultados de una investigación acerca de la implementación del Modelo Pedagógico por Alternancia en Petén, Guatemala, que tuvo como propósito determinar la influencia del modelo en el desarrollo local. El enfoque es mixto, no experimental, explicativo, utilizando instrumentos que permitieron el análisis de estadística inferencial y el empleo de métodos etnográficos. El diseño del muestreo fue bietápico, direccionado a estudiantes, docentes y directores. Con los resultados de la investigación se puede afirmar que este modelo está contribuyendo con el desarrollo humano mediante los procesos de educación sistemática y formación para la vida en el que participan los jóvenes que en el futuro pueden convertirse en los principales actores para el desarrollo del medio rural.

\section{Palabras clave:}

Educación. Modelo Pedagógico. Alternancia. Medio rural. Desarrollo local.

\section{Lisichiku limaykuna:}

yaćhana, yaćhachiku

likapaana, hukninkaynin, kikinmalkap wiñaynin.

\section{Nibarintsi katingaro:}

Jigotagantsi okanta kaninasati. Okanta jigotagantsi kara jaantagitepage imagantsipagekë.

Datos de la autora

Deysi Lisbeth Rodriguez Max, es Docente e investigadora de la Universidad de San Carlos de Guatemala, Magíster en Desarrollo Rural, Licenciada en Pedagogía y Ciencias de la Educación, estudiante del Doctorado en Investigación para el Desarrollo Social en el Centro Universitario de Sur Oriente de la Universidad de San Carlos de Guatemala. 


\section{Influence of the Alternation Pedagogical Model in the Local Development of Five Rural Communities in the Department of Petén, Guatemala}

\begin{abstract}
This article presents the results of an investigation about the implementation of the by Alternation Pedagogical Model in Petén, Guatemala, whose purpose was to determine the influence of the model on local development. The approach is mixed, not experimental, explanatory, using instruments that allowed the analysis of inferential statistics and the use of ethnographic methods. The sampling design was two-stage, aimed at students, teachers and principals. With the results of the research, it can be affirmed that this model is contributing with human development through the processes of systematic education and training for life in which young people participate, which in the future may become the main actors for the development of the environment rural.
\end{abstract}

\section{Keywords}

Education. Pedagogical model. Alternation.

Rural environment. Local development.

\section{Influência do modelo pedagógico por alternância no desenvolvimento local de cinco comunidades rurais do departamento de Petén, Guatemala}

\section{Resumo}

Este artigo apresenta os resultados de uma pesquisa sobre a implantação do Modelo Pedagógico por alternância em Petén, Guatemala, cujo propósito foi determinar a influência do modelo no desenvolvimento local. A abordagem é mista, não experimental, explicativa, utilizando instrumentos que permitiram a análise de estatísticas inferenciais e a utilização de métodos etnográficos. O desenho amostral foi em duas etapas, destinado a estudantes, professores e diretores. Com os resultados da pesquisa, pode-se afirmar que este modelo está contribuindo com o desenvolvimento humano através dos processos de educação sistemática e formação para a vida em que participam os jovens, que no futuro podem se tornar os principais atores para o desenvolvimento do meio ambiente. rural.
Palavras-chave:

Educação, modelo pedagógico, alternância, meio rural, desenvolvimento local. 


\section{Introducción}

La educación es la clave para el progreso de todo ser humano y del medio en donde se desenvuelve, y el desarrollo a la vez, constituye un cambio favorable de las personas que viven en sociedad, en ese sentido, como una alternativa de educación sistemática y desarrollo, surgen los Núcleos Familiares Educativos para el Desarrollo (NUFED), centros educativos del ciclo básico del Ministerio de Educación (MINEDUC) de Guatemala, basados en el modelo pedagógico por alternancia, "como una alternativa para los jóvenes del área rural para ofrecer una formación y capacitación para el trabajo a través de una educación integral" (MINEDUC, 2010, p. 51).

Este artículo parte de la investigación acerca de la Influencia del modelo pedagógico por alternancia en el desarrollo local de cinco comunidades rurales del departamento de Petén, Guatemala, ante la necesidad de conocer la efectividad del funcionamiento de los NUFED, un programa establecido por el Ministerio de Educación mediante convenios con otros países para contribuir con la educación en el medio rural. También hace énfasis en la experiencia del modelo en países como Perú, Brasil, Argentina, República Dominicana y otros.

El estudio aporta elementos para la reflexión institucional en aras de fortalecer el funcionamiento de los NUFED, y la formación por alternancia que hacen un significativo aporte al impulso de la educación de los jóvenes del área rural, en donde su desarrollo está asociado también al de sus familias y comunidades, y a la luz de sus resultados, se pueda encontrar un punto de partida para la implementación de acciones efectivas para lograr el desarrollo rural en Petén, Guatemala.

\section{Los centros educativos de formación por alternancia}

De acuerdo con Puig (2006) un Centro Educativo de Formación por Alternancia (CEFFA) es una asociación de familias, personas e instituciones que buscan solucionar una problemática común, de evolución y de desarrollo local a través de actividades de formación, principalmente de jóvenes sin incluir a los adultos.

\section{Los Núcleos Familiares Educativos para el Desarrollo}

Para el MINEDUC (2010) los NUFED son centros educativos del nivel medio, ciclo básico del Ministerio de Educación, que junto a las familias y otras personas buscan solucionar una problemática común, de evolución y de desarrollo local a través de actividades de educación y formación. Pertenecen a un programa oficial del Ministerio de Educación, de la Dirección General de Educación Extraescolar, creado como alternativa de educación y desarrollo personal, familiar y comunal, para jóvenes y señoritas facilitándoles una formación integral en los tres grados del ciclo de educación básica del nivel medio.

Según Menéndez (2002) los NUFED tienen como propósito fundamental proporcionar a los jóvenes del área rural, egresados de la escuela primaria, una formación general basada en las necesidades, problemas e inquietudes de la población. Esta formación está asociada a una 
preparación profesional, a la cual se agrega la educación equivalente a los tres años del ciclo básico de educación media. El proceso de formación de los centros comienza con un estudio minucioso del entorno, que se efectúa con la participación de todos los vecinos, autoridades y alumnos potenciales.

A raíz del terremoto que ocurrió en Guatemala en febrero de 1976, nacen los Núcleos Familiares Educativos para el Desarrollo, como parte de la cooperación externa de países como Francia, para la reconstrucción del país se aperturaron algunos centros enfocados desde ese entonces principalmente al trabajo en las zonas rurales en el Altiplano de Guatemala, donde han apoyado a las comunidades por medio de la atención en la educación básica (Figueroa, 2010). El primer NUFED se creó en el año de 1978 en la aldea de San José Chirijuyú, Tecpán, Chimaltenango; $y$ en Petén el primer centro fue el NUFED número 4 en la Cooperativa Flor de la Esperanza de La Libertad en 1980, cerrando sus servicios por efectos del conflicto armado ocurrido en Guatemala (A. Constancia, comunicación personal, 11 de mayo 2014) al año 2013 el MINEDUC ha creado 622 centros a nivel nacional, 60 en Petén, atendiendo más de 138,000 estudiantes a nivel nacional y 25,543 en Petén (MINEDUC, 2013). Entre sus bases legales se pueden citar el Convenio No. 3-77 de fecha 29 de junio de 1977 de cooperación entre la Embajada de Francia y el MINEDUC, Acuerdo Ministerial 869-83 de fecha 14 de noviembre de 1983; Acuerdo Ministerial 3596-2011 que autoriza el Currículo Nacional Base con la modalidad de alternancia (Arizmendy y Escobedo, 2013).

\section{La importancia de la educación para el medio}

\section{rural}

La educación es un proceso importante mediante el cual el ser humano adquiere conocimientos, valores, costumbres, formas de actuar y de ser que le permite desenvolverse en la vida. Para Aldana(2004), "la educación es el proceso de influencias que determinan o transforman el modo de ser, pensar, sentir y actuar de los seres humanos" (p. 54), y juega un papel decisivo en el desarrollo, ya que puede ayudar a concienciar a cerca de la necesidad de reconducir los patrones de crecimiento y progreso sobre los principios de sostenibilidad, equidad y compromiso social, dejando a un lado los clásicos criterios economicistas, materialistas e individualistas que han caracterizado tradicionalmente dicho concepto (Martínez, 2010).

La educación que imparten los NUFED es una práctica de la educación profesional, porque busca que los estudiantes puedan formarse y aprender un oficio a través de un proceso de auto formación que tiene como objetivo insertar al estudiante en el mundo laboral, social y profesional con un modelo pertinente. Es necesario resaltar que, el acceso al ciclo básico es más limitado para mujeres, pueblos indígenas y residentes en el área rural, ya que un $53 \%$ de hombres y un $47 \%$ de mujeres tienen acceso al ciclo básico, un $75 \%$ de no indígenas y un $25 \%$ de indígenas, un 65\% del área urbana y un 39\% del área rural (Ortega y Avendaño, 2012).

Para el Centro de Estudios para el Desarrollo Rural CESDER (1998) un buen punto de partida para la comprensión de la situación de la educación rural es el reconocimiento de una profunda crisis educativa rural. Los campesinos de América Latina son más pobres hoy que hace veinte o treinta años, la mayoría de los países de la región están en las zonas rurales; el deterioro ecológico avanza a pasos agigantados, las culturas rurales, múltiples y diversas, están siendo desmanteladas a la misma velocidad y, no pocas veces, la educación tradicional ha contribuido a la producción de todo esto. 
En el caso específico del departamento de Petén Schwartz (2010) afirma que la sociedad petenera ha evolucionado grandemente a través de los últimos 50 años, sin embargo, la condición social y económica del sector rural no ha cambiado dramáticamente a través de los años. Un dato importante que menciona Schwartz es que, en 1960 Petén no tenía una infraestructura moderna como hoy, la mayoría de la gente era pobre, pero todo mundo tenía para vivir y comer, y más tiempo para compartir en sociedad, por lo que hace énfasis en que todo desarrollo tiene también un grado de pérdida y consecuencias, y que la modernidad en sí no es desarrollo.

La gente del campo es en su mayoría pobre y depende de la agricultura de subsistencia, por lo que el desarrollo rural afronta el reto de reducir la pobreza y un instrumento para tal fin es ampliar la cobertura educativa a la mayor parte de la población. Según Bonilla (2012) en el mundo globalizado, los conocimientos y la educación han pasado a ser los factores más determinantes para que los productores rurales puedan desenvolverse y mantenerse al menos sobreviviendo.

En el área rural de Guatemala muchos niños y niñas abandonan el ciclo escolar para apoyar a sus padres en las tareas de cultivos, no obstante, los ingresos son muy bajos y no toman conciencia de la importancia de tener una mayor y mejor educación, podrían tener mejores oportunidades laborarles. En este caso, la educación con el modelo pedagógico por alternancia brinda esta oportunidad a los jóvenes de poder hacer prácticas agrícolas y educación formal a la vez, trabajar una semana en la escuela y en el campo.

\section{La Pedagogía y el Modelo Pedagógico de Alternancia en Guatemala}

Para Aldana, el origen de la palabra "se encuentra en las siguientes voces griegas: paidos que significa niño, y agogía, que significa conducir, por lo que la palabra pedagogía etimológicamente significa conducción del niño" (2004, p. 101). Otros autores lo definen como el estudio intencionado, sistemático y científico de la educación; es decir, la disciplina que tiene por objeto el planteo, estudio y solución del problema educativo, el conjunto de normas, principios y leyes que regulan el hecho educativo sistemático (Lemus, 2007). Desde el punto de vista de la pedagogía crítica, Aldana (2004) refiere que, es la que vincula la educación con el mundo real, también es popular y va al encuentro de experiencias educativas y su principal interés es hacer de la educación una herramienta y una función al servicio de todo aquello que transforme la vida de un pueblo.

El Modelo Pedagógico por Alternancia, nace antes de la Segunda Guerra Mundial, entre 1935 y 1937 en una Provincia de Francia en la pequeña aldea de Sérignac Péboudou, en donde campesinos liderado por Jean Peyrat y el sacerdote Granerau reflexionaban acerca de la educación para los jóvenes en el área rural y crean la primera Maisons Familiares Rurales (MFR) en Lauzun en el año de 1937 (MINEDUC, 2010).

La génesis de la metodología surge por la necesidad de un grupo de jóvenes en trabajar y estudiar, dado que la escuela tradicional no respondía a sus expectativas, se creó una escuela en la que los alumnos pasaran una temporada en ella y otra con la familia; ambos momentos se constituirían en espacios de formación vinculados a su propio medio, diseñándose la estructura de la pedagogía de la alternancia. A partir de 1946 se empieza a hablar establemente de ésta, y poco después comienza el interés de destacados pedagogos por el modelo (MINEDUC, 2010). 
Según el MINEDUC (2010) después del terremoto del 4 de febrero de 1976 en Guatemala, que afectó principalmente a familias campesinas e indígenas del área rural, surgen una serie de proyectos para cubrir las necesidades básicas de las comunidades, entre ellas la educación como parte de la reestructuración del país, para responder a las necesidades, se crea un programa administrado por la misma comunidad con las características socioeconómicas y culturales de cada población. Es así como en Guatemala, en el año de 1978 se creó el primer centro del Programa Nacional de los Núcleos Familiares Educativos para el Desarrollo con la aplicación de la modalidad de la alternancia en la aldea San José Chirijuyú, Tecpán, departamento de Chimaltenango, como resultado del Convenio 3-77 firmado entre el Ministerio de Educación y la Embajada de Francia acreditada en el país, el 29 de junio de 1977, aprobado por Acuerdo Gubernativo No. 026 de fecha 27 de junio de 1978.

El Ministerio de Educación en el Acuerdo Ministerial Número 3596-2011 establece que, la alternancia en los NUFED se organiza en permanencias en el medio de vida, familia empresa o grupos sociales; y permanencias estadías en los centros educativos, las que podrán variar de acuerdo a las necesidades de los estudiantes y su medio, conforme al calendario ocupacional o ciclos productivos y naturales de las regiones, y su articulación con el Currículum Nacional Base (MINEDUC, 2010).

\section{Los NUFED una alternativa para el desarrollo en el medio rural}

Para Valenciano y Gómez (2001) el mundo rural puede definirse como "el conjunto de regiones o zonas con actividades diversas: la agricultura, la artesanía, la pequeña y mediana industria, el comercio, servicios y otros" (p. 151), en las que se asientan, pueblos, aldeas, pequeñas ciudades y centros regionales, así como espacios naturales y cultivados.

El medio rural se encuentra hoy en un frágil equilibrio ante las funciones que se le exigen, por un lado, se le pide que produzca alimentos y facilite materias primas en el marco de un desarrollo sostenible. Por otro, que sea zona para el equilibrio ecológico y para las actividades de ocio y descanso. La población en el medio rural muchas veces es escasa, no obstante, con el crecimiento demográfico por lo general estos asentamientos pasan a formar parte del mundo urbano (Valenciano y Gómez, 2001).

De acuerdo con García (2009) la alternancia que se imparte en los NUFED es un verdadero sistema de formación que se construye partiendo de la hipótesis de que los saberes locales pueden generar progreso en ámbitos más allá de lo local, en este contexto, el tema de los valores es muy sensible. Así, el contrapunto esperado a los modos de hacer muy centrados en el crecimiento meramente económico, es el del desarrollo de las personas.

Algunos autores indican que existen Organizaciones que trabajan para la educación, y al referirse sobre la implementación del modelo pedagógico de alternancia refieren que:

Manejar con éxito el modelo pedagógico de alternancia supone diseñar un plan de formación pertinente, es decir, entrelazar continuamente el currículo con el medio, para integrar el currículo oficial con la compleja realidad del joven en formación. Lo que se espera como resultados de este modelo es que los egresados sean líderes locales que vivan dig- 
namente de su trabajo y en su territorio; jóvenes capaces de emprender proyectos que contribuyan a su desarrollo personal y familiar para conseguir así el progreso de toda la comunidad (García, 2009).

Este planteamiento pedagógico supone que el joven sea responsable de su propio conocimiento, de la búsqueda de soluciones a los problemas, y de la puesta en marcha de proyectos. Se trata de convertir esos proyectos en una oportunidad de aprender a emprender; los jóvenes, que son actores locales de desarrollo y pequeños empresarios, adquieren la capacidad de emprender proyectos de mejora que responden a las necesidades u oportunidades locales y que facilitan el empleo o autoempleo; en cualquier caso, siempre habrá una pequeña contribución significativa al alivio de la pobreza y al desarrollo local.

\section{Experiencias de la formación por alternancia en diversos países}

De acuerdo con García (2009), ya existen experiencias sobre los centros educativos por alternancia en diferentes países, entre los cuales se pueden citar a:

Guatemala: la experiencia se basa en la expansión del modelo de la alternancia, mediante la firma de convenio entre Guatemala y con la Embajada de Francia en Guatemala para implementar el programa mediante los NUFED, hoy existen más de seiscientos centros, sin embargo, su desafío principal es la sostenibilidad económico financiera del modelo.

En Argentina: los centros educativos de formación por alternancia llegan en la década de 1970 , en la actualidad hay más de cien escuelas de alternancia, y en los logros se encuentran el desarrollo de tres redes de escuelas de alternancia con amplia implantación en el país, el reconocimiento de la pedagogía y su financiación.

En Colombia: la experiencia se basa en la calidad de la pedagogía de la alternancia y los resultados académicos de los egresados, los Bachilleres con modalidad empresarial y agropecuaria, obtiene puntajes representativos y se ubica en la categoría de nivel alto en la comparativa nacional.

En Perú: la experiencia se basa en la pertinencia de la pedagogía de la alternancia en ámbitos rurales y los proyectos de los egresados, un punto interesante es que, en el 2001 ProRural y dos organizaciones de la sociedad civil peruana, presentaron ante el Ministerio de Educación una propuesta para la implementación del sistema de educación en alternancia, ahora los jóvenes egresados ponen en marcha proyectos profesionales que les permiten ganarse la vida y elevar el nivel de sus familias y sus comunidades; partiendo de una pequeña inversión inicial con el acompañamiento de las familias y los monitores, se pueden observar logros en la mejora de la calidad de vida como.

En Brasil: los CEFFA comenzaron en 1968, hoy hay más de 250 escuelas en 17 Estados y en 1977 comenzó a funcionar el Equipo Pedagógico Nacional con un responsable pedagógico de cada Regional, uno de sus logros esenciales es que la mayoría de los monitores ha finalizado la formación pedagógica inicial, los jóvenes culminan con un proyecto profesional y se insertan profesionalmente a través de los créditos de jóvenes. 
En Uruguay: las primera Escuelas Familiares de Alternancia (EFA) comienzan en 1980, recientemente se reconoció la pedagogía de alternancia como metodología de enseñanza para el Ciclo Básico y en alumnos con edades entre los 12 a 15 años, y a raíz de un seminario internacional celebrado en el 2005 se reconoció la alternancia en algunos cursos de formación profesional para jóvenes entre 15 a 18 años con orientación agrícola y ganadera, y se aprobó una ley que otorga un subsidio anual para las EFA.

República Dominicana: se han establecido ocho escuelas distribuidas en todo el país y se ha constituido la unión de escuelas con apoyo internacional y la formación de monitores para adoptar la metodología de trabajo, un logro importante has sido la graduación de alumnos del sector agrícola con reconocimiento del Ministerio de Educación como Bachiller Técnico en Alternancia.

\section{Metodología}

Para este estudio se utilizó un enfoque mixto basado en una metodología cualitativa y cuantitativa, la parte cualitativa mediante la evidencia etnográfica para verificar la implementación de la metodología por alternancia, condiciones de los centros educativos, observación en la comunidad, y grupos focales con directores de los NUFED de Petén. La parte cuantitativa se llevó a cabo a través de la aplicación de encuestas a los docentes y estudiantes; por su nivel de alcance la investigación fue de tipo explicativo y su diseño no experimental.

\section{La hipótesis y las variables}

\section{Hipótesis}

El modelo pedagógico por alternancia que se implementa en los NUFED tiene una influencia importante en el desarrollo local en la aldea El Naranjo de Flores, Caserío Salvador Fajardo de la Libertad, Caserío San Valentín Las Flores de San Francisco, Barrio San Ramón y Caserío El Naranjón de Dolores, Petén.

\section{Variable Independiente}

La implementación del modelo pedagógico por alternancia de los NUFED.

\section{Variable Dependiente}

Características de desarrollo local en cinco comunidades rurales ubicadas en los municipios de Flores, San Francisco, La Libertad y Dolores Petén. 
Tabla 1

Definición operacional de las variables

\begin{tabular}{|c|c|c|}
\hline Variables & Indicadores & Instrumentos \\
\hline $\begin{array}{l}\text { La } \\
\text { implementación } \\
\text { del modelo } \\
\text { pedagógico por } \\
\text { alternancia. }\end{array}$ & $\begin{array}{l}\text { - Condiciones de la escuela: físicas, recursos materiales, } \\
\text { financieros y humanos. } \\
\text { - Horario de clases. } \\
\text { - Sostenibilidad económica del NUFED. } \\
\text { - Sistema de monitoreo. } \\
\text { - Características de los monitores: profesión, nivel } \\
\text { académico, especialidad en cursos técnicos y } \\
\text { experiencia docente. } \\
\text { - Participación de los padres } \\
\text { - Capacitación del recurso humano. }\end{array}$ & $\begin{array}{l}\text { Encuesta a docentes. } \\
\text { Encuesta a } \\
\text { estudiantes. }\end{array}$ \\
\hline & $\begin{array}{l}\text { - Desarrollo de la metodología. } \\
\text { - Plan de formación y el CNB } \\
\text { - Aplicación de cursos técnicos } \\
\text { - Prácticas de alternancia. } \\
\text { - Pertinencia con los objetivos del modelo y necesidades } \\
\text { de las comunidades. }\end{array}$ & $\begin{array}{l}\text { Guía de Observación } \\
\text { y fichas de campo. }\end{array}$ \\
\hline $\begin{array}{l}\text { Características } \\
\text { de desarrollo } \\
\text { local en cinco } \\
\text { comunidades } \\
\text { rurales ubicadas } \\
\text { en los municipios } \\
\text { de Flores, San } \\
\text { Francisco, La } \\
\text { Libertad y Dolores } \\
\text { Petén }\end{array}$ & $\begin{array}{l}\text { - Evidencia de proyectos. } \\
\text { - Evidencia de desarrollo personal } \\
\text { - Existencia de huertos escolares y parcelas } \\
\text { demostrativas. } \\
\text { - Oportunidad y ocupación laboral de los jóvenes } \\
\text { egresados. } \\
\text { - Percepción de Directores, docentes, estudiantes, } \\
\text { acerca de la influencia del modelo por alternancia en } \\
\text { el desarrollo local. } \\
\text { - Inserción laboral de los jóvenes. }\end{array}$ & $\begin{array}{l}\text { Grupo focal con } \\
\text { directores de los } \\
\text { NUFED de Petén. }\end{array}$ \\
\hline
\end{tabular}

Nota: Datos sistematizados para la operacionalización de las variables del estudio.

\section{Población}

La población universo estuvo conformada por todos los Núcleos Familiares Educativos para el Desarrollo (NUFED) que existen en el departamento de Petén, que están localizados en los ocho municipios siguientes: Flores, San Andrés, La Libertad, San Francisco, Santa Ana, Dolores, Sayaxché y Melchor de Mencos, Petén.

\section{La muestra}

Se utilizó el diseño de muestreo bietápico para la selección de los municipios y las comunidades, de forma aleatoria simple se seleccionó a los municipios que tienen NUFED, posteriormente, de forma direccionada se seleccionó a las cinco comunidades rurales, utilizando el criterio con ocho a diez años de funcionamiento. Las unidades muestrales fueron los Núcleos Familiares ubicadas en cinco municipios, y las unidades de análisis los docentes y estudiantes de tercero básico por estar en la fase final de formación y el 63\% de directores de los NUFED del departamento de Petén. 
Tabla 2.

La Muestra del Estudio.

\begin{tabular}{lllcc} 
No. & Municipio & \multicolumn{1}{c}{ NUFED } & Docentes & $\begin{array}{c}\text { Estudiantes de } \\
\text { tercero básico }\end{array}$ \\
\hline $\mathbf{1}$ & Flores & Aldea el Naranjo No. 429. & 5 & 26 \\
$\mathbf{2}$ & Dolores & Barrio San Ramón No. 9. & 10 & 31 \\
\hline $\mathbf{3}$ & Dolores & Caserío el Naranjón No. 55 & 3 & 10 \\
$\mathbf{4}$ & San Francisco & $\begin{array}{l}\text { Caserío San Valentín Las Flores No. } \\
\text { 38. }\end{array}$ & 10 & 19 \\
\hline $\mathbf{5}$ & La Libertad & Caserío Salvador Fajardo No. 160. & 3 & 13 \\
\hline & & Total & 31 & 99 \\
\hline
\end{tabular}

Nota: Los datos son proporcionados por el Ministerio de Educación de Guatemala, año 2013.

\section{Métodos e instrumentos de recolección de los datos}

Para lograr el objetivo del estudio y con base en su diseño, se utilizaron diversos instrumentos de colecta de datos como: encuesta estructurada para docentes y estudiantes, guía de observación para conocer las condiciones de funcionamiento de los centros, guía para grupo focal con los directores para la obtención y contraste de la información, y documentación para la fundamentación del estudio.

\section{Tratamiento de los datos}

La información cuantitativa se derivó de las encuestas y se analizaron a través de un tratamiento estadístico descriptivo e inferencial de la siguiente manera: para el tratamiento y análisis de la información se organizó la información a través de bases de datos desagregadas e integradas en Microsoft Excel, se hizo el trabajo de análisis de la información utilizando el Software IBM SPSS Statistics 20, se elaboraron figuras y tablas estadísticas, se interpretaron y discutieron los resultados. Para el tratamiento de la información cualitativa se tomó en cuenta los instrumentos utilizados en el grupo focal con directores, las guías de observación, las fichas de campo y fotografías tomadas durante el estudio.

\section{Resultados}

Los resultados de docentes y estudiante indican que los NUFED funcionan en jornada doble, no obstante, se pudo observar que lo hacen únicamente en la semana de estadía en el aula, no así en la semana de alternancia. La proporción de NUFED que trabajan en jornada doble (77.8\%) es significativamente mayor que los que trabajan una jornada (22.2\%), Chi Cuadrado de Pearson 49.356 (8gl) p<.001. De las condiciones de funcionamiento, todos cuentan con edificio propio y la infraestructura es buena, la mayoría tiene servicios básicos como: energía eléctrica, agua potable, solo dos centros no tienen ninguno de los servicios básicos. 
Tabla 3.

Condiciones funcionamiento de los NUFED.

\begin{tabular}{|c|c|c|c|c|c|c|c|}
\hline NUFED & $\begin{array}{l}\text { Edificio } \\
\text { Propio }\end{array}$ & $\begin{array}{l}\text { Energía } \\
\text { eléctrica }\end{array}$ & $\begin{array}{l}\text { Agua } \\
\text { potable }\end{array}$ & Sanitario & Terreno & $\begin{array}{l}\text { Recursos } \\
\text { materiales }\end{array}$ & $\begin{array}{c}\text { Área } \\
\text { recreativa }\end{array}$ \\
\hline $\begin{array}{l}\text { Aldea el Naranjo No. } \\
\text { 429. Aldea El Naranjo } \\
\text { de Flores. }\end{array}$ & $X$ & $X$ & $X$ & $X$ & $X$ & $X$ & $X$ \\
\hline $\begin{array}{l}\text { Barrio San Ramón No. } \\
9 \text { de Dolores. }\end{array}$ & $x$ & $X$ & $X$ & $X$ & $X$ & $X$ & $X$ \\
\hline $\begin{array}{l}\text { Caserío el Naranjón } \\
\text { No. } 55 \text { de Dolores. }\end{array}$ & $X$ & o & o & 0 & o & 0 & o \\
\hline $\begin{array}{l}\text { Caserío San Valentín } \\
\text { Las Flores No. } 38 \text { de } \\
\text { San Francisco. }\end{array}$ & $X$ & $X$ & $X$ & $X$ & $X$ & $X$ & $X$ \\
\hline $\begin{array}{l}\text { Caserío Salvador } \\
\text { Fajardo No. } 160 \text { de La } \\
\text { Libertad. }\end{array}$ & $X$ & 0 & 0 & $X$ & 0 & 0 & $x$ \\
\hline
\end{tabular}

Nota: Datos obtenidos del trabajo de campo, en donde $\mathrm{X}=$ Presencia de la variable, $\mathrm{O}=\mathrm{Au}-$ sencia de la variable.

Respecto a los recursos, la proporción de estudiantes que indicó que los docentes utilizan únicamente pizarrón y marcador (36.4) es mayor que los que indicaron que utilizan herramientas propias de la alternancia. Chi Cuadrado de Pearson 78.973 (28gl) p<.001. La figura 5 muestra el desarrollo de la metodología que emplean los docentes, como la formación general y puesta en común, el cuaderno de la realidad y la guía de estudio, el menor porcentaje indicó que reciben la charla profesional.

\section{Figura 1.}

Implementación de instrumentos de la alternancia

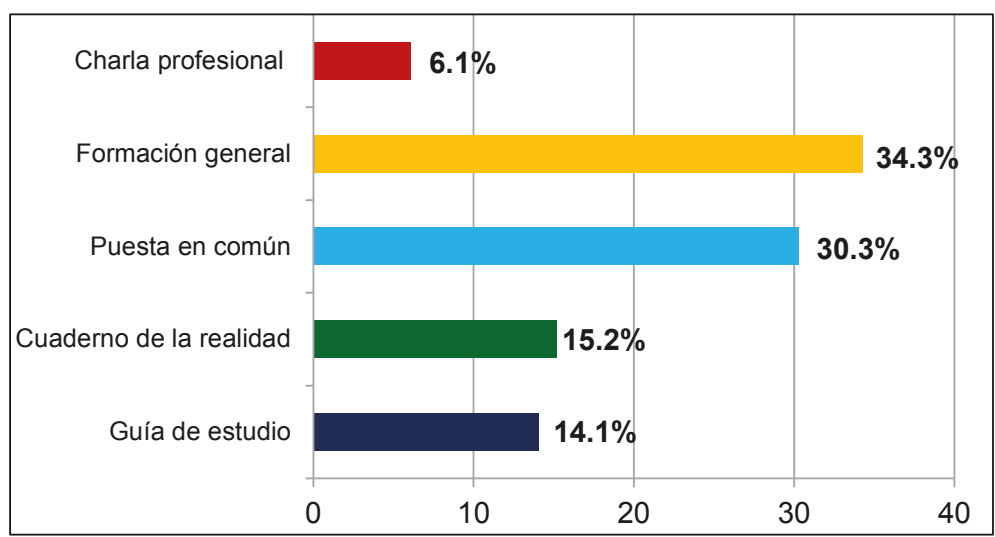

Nota: Datos obtenidos mediante el trabajo de campo el estudio. 
Por otra parte, los resultados también indican que la proporción de los NUFED que trabajan en el aula (63.6\%) es mayor que los que trabajan en el campo (1.0\%) y de los que trabajan en el aula y campo (35.4\%), Chi Cuadrado de Pearson 35.893 (8gl) p<.001. De igual forma, el mayor porcentaje de estudiantes de las cinco comunidades indicó que siempre visitan las parcelas demostrativas, en proporciones equitativas del $23 \%$ indicaron que es una vez al mes y cada tres días, sin embargo, un $16 \%$ indicó que nunca visitan las parcelas ya que hay centros que no implementan ninguna actividad de alternancia.

los tipos de proyectos que más se implementan son los huertos; estos se trabajan tanto en las prácticas escolares como en las viviendas de los estudiantes o huertos familiares. De acuerdo con los resultados, la proporción tanto de estudiantes como de docentes que indicaron que trabajan huertos es estadísticamente mayor (68\% y 59\%) que los que indicaron que trabajan otro tipo de proyectos. Chi Cuadrado de Pearson 69.086 (16gl) p<.oo1.

Figura 2.

Tipo de proyectos que realizan los estudiantes.

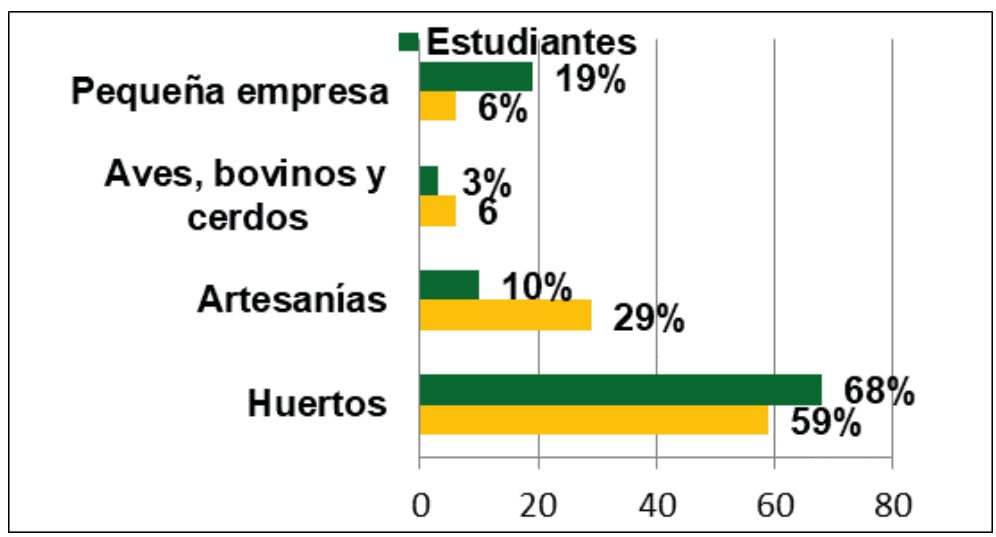

Nota: Datos obtenidos mediante el trabajo de campo el estudio.

En el estudio también se encontró que la proporción de estudiantes que ha continuado implementando el área agrícola es mayor (77.4\%) que la proporción de estudiantes que tienen una pequeña empresa o granja de animales Chi cuadrado de Pearson 31.560 (12gl) p<.001.

La figura 11 muestra que, entre los cultivos que más desarrollan los estudiantes como parte de trabajo agrícola se pueden mencionar las especies: los granos básicos (maíz, frijol y arroz); las verduras (rábano, berenjena, chile dulce, ayote y hierbas) y las frutas (naranja, limón y papaya). Un dato importante es que, los productos que se consumen en la comunidad provienen principalmente de los huertos familiares, escolares, y tiendas locales. 
Figura 3.

Procedencia de los productos que se consumen en la localidad.

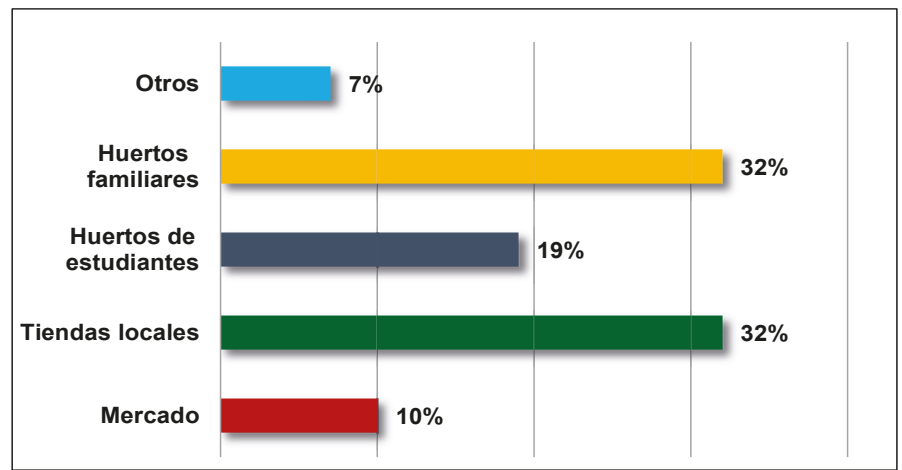

Nota: Datos obtenidos mediante el trabajo de campo el estudio.

La mayoría de los docentes considera que el modelo por alternancia contribuye al desarrollo comunitario y que el mayor beneficio es la generación de ingresos económicos a las familias a través del aporte de los jóvenes, la existencia de nuevos profesionales en la comunidad y oportunidad de educación.

Figura 4.

Contribución del modelo por alternancia en el desarrollo local

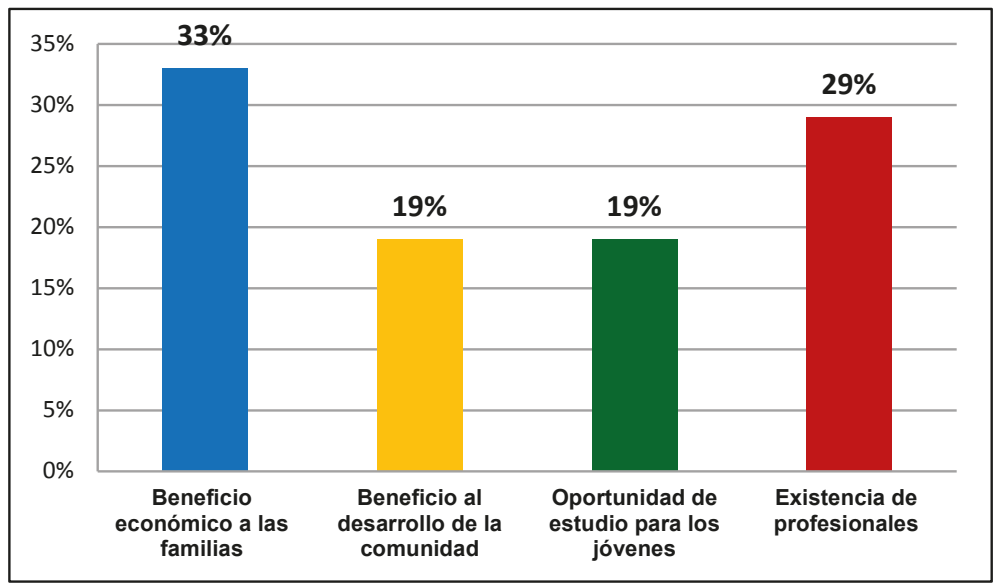

Nota: Datos obtenidos mediante el trabajo de campo el estudio.

De acuerdo a la versión de los docentes en base a su experiencia en las comunidades, los estudiantes que han egresado de los NUFED realizan diversas actividades, estadísticamente se pudo comprobar que la proporción de estudiantes que continúan estudios en el Ciclo Diversificado (45.2\%) es mayor que la proporción de los jóvenes que se quedan trabajando la agricultura en la comunidad (8.3\%), Chi Cuadrado de Pearson 51.42 (20gl) p< .001. 
Tabla 4.

Ocupación de egresados de los NUFED, datos de estudiantes.

\begin{tabular}{|l|c|c|c|c|c|c|c|}
\hline \multicolumn{1}{|c|}{ NUFED } & Agricultura & $\begin{array}{c}\text { continúan } \\
\text { estudiando }\end{array}$ & $\begin{array}{c}\text { Trabajan y } \\
\text { estudian }\end{array}$ & desempleados & $\begin{array}{c}\text { Tienen } \\
\text { una } \\
\text { profesión }\end{array}$ & $\begin{array}{c}\text { Emigraron } \\
\text { a Estados } \\
\text { Unidos }\end{array}$ & $\begin{array}{c}\text { Tienen } \\
\text { un } \\
\text { trabajo }\end{array}$ \\
\hline $\begin{array}{l}\text { Barrio San } \\
\text { Ramón }\end{array}$ & $6.5 \%$ & $77.4 \%$ & $12.9 \%$ & $0.0 \% \%$ & $3.0 \%$ & $0.0 \%$ & $3.2 \%$ \\
\hline $\begin{array}{l}\text { Caserío San } \\
\text { Valentín Las } \\
\text { Flores }\end{array}$ & $10.5 \%$ & $52.6 \%$ & $15.8 \%$ & $0.0 \%$ & $5.1 \%$ & $0.0 \%$ & $21 \%$ \\
\hline $\begin{array}{l}\text { Caserío El } \\
\text { Naranjón }\end{array}$ & $0.0 \%$ & $20.0 \%$ & $30.8 \%$ & $0.0 \% \%$ & $0 \%$ & $40.0 \%$ & $0.0 \%$ \\
\hline $\begin{array}{l}\text { Caserío } \\
\text { Salvador } \\
\text { Fajardo }\end{array}$ & $15.4 \%$ & $23.1 \%$ & $15.4 \%$ & $0.0 \% \%$ & $0 \%$ & $0.0 \%$ & $23.1 \%$ \\
\hline $\begin{array}{l}\text { Aldea El } \\
\text { Naranjo }\end{array}$ & $11.5 \%$ & $34.6 \%$ & $11.5 \%$ & $11.5 \% \%$ & $1.0 \%$ & $0.0 \%$ & $7.7 \%$ \\
\hline Total & $9.1 \%$ & $48.5 \%$ & $15.2 \%$ & $3.0 \%$ & $9.1 \%$ & $5.1 \%$ & $10.0 \%$ \\
\hline
\end{tabular}

Nota: Datos obtenidos mediante el trabajo de campo el estudio.

Es evidente que la proporción de estudiantes que continúan estudios en el ciclo diversificado o en el nivel superior (48.5\%) es mayor que la proporción de los jóvenes que se dedican al trabajo agrícola (9.1\%) o con los desempleados (3\%), Chi Cuadrado de Pearson 77.626 (32gl) $\mathrm{p}<.001$.

Respecto a los resultados del grupo focal, los directores indicaron que las principales fortalezas que tienen los NUFED son: brindar a los jóvenes de escasos recursos la oportunidad para continuar estudios del ciclo básico; la capacidad de formar a los estudiantes para la vida a través de la enseñanza de un oficio y fortalecer el medio de vida y por lo consiguiente, contribuir con el desarrollo comunitario, aún con las debilidades que presentan ante la falta de recursos y acompañamiento para la implementación del modelo pedagógico.

Sin embargo, también manifestaron que, "Los NUFED están abandonados, como directores tenemos que ver que hacemos para poder trabajar la alternancia y solventar las diferentes necesidades del establecimiento". Por lo que consideran que es difícil implementar el modelo en su totalidad debido a que no se cuenta con los recursos adecuados ni con los docentes para impartir las clases que requieren de una especialidad. Los directores manifestaron que no hay compromiso por parte de las autoridades, de los docentes, ni de los padres de familia, porque muchas veces no permiten que los hijos Ileguen en la semana de alternancia porque tienen que trabajar en las tareas del hogar o en algún lugar en donde reciben un salario.

\section{Discusión}

El modelo por alternancia constituye una alternativa para promover el desarrollo personal, familiar y del medio en que viven los jóvenes del área rural, es por ello que el estudio 
estuvo orientado en determinar la influencia del modelo en el desarrollo, en el medio rural de Petén, Guatemala, no obstante, para lograr su efectividad, es necesario hacer un análisis acerca de todos los factores asociados con la correcta implementación del modelo por alternancia y los NUFED, en ese sentido, Chavén (2007) refiere que, los NUFED fueron creados para proporcionar a los jóvenes del área rural, una formación del ciclo básico, basado en las necesidades y problemas asociadas a una preparación profesional, por lo tanto, deben tener las condiciones adecuadas para brindar una educación que responda a los intereses de la población en el área rural.

Los resultados de este estudio explican con claridad que los NUFED no están recibiendo el apoyo necesario para su buen funcionamiento, aunque tienen edificio propio, no todos cuentan con los servicios básicos y recursos propios del modelo para su funcionamiento, ante tal situación, las autoridades educativas deben atender de una mejor manera a los centros educativos con los recursos necesarios, pero por otra parte, los directores deben ser agentes gestores para suplir las necesidades de acuerdo al modelo; se encontró que los centros no están recibiendo el acompañamiento técnico pedagógico por parte del Ministerio de Educación, por lo tanto, con el programa sólo están ampliando la cobertura del ciclo básico en el medio rural, sin cumplir con la filosofía del modelo por alternancia.

Como se resalta en los fundamentos teóricos de este estudio, el modelo por alternancia consiste en la formación teórica y práctica, tal como lo definen algunos autores, se debe hacer alternar períodos de trabajo con su familia, en la empresa o parcela familiar, y períodos de reflexión y capacitación en el centro de estudio (Chavén, 2007) no obstante, mediante los métodos de encuesta y etnográficos se encontró que el modelo no se está implementando en su totalidad. Se comprobó que en la mayoría de los centros no se están empleando todos los instrumentos propios de la alternancia, aunque se realizan algunas actividades en el campo, sólo en un centro se observó el uso de los cuadernos de visitas domiciliarias.

Con relación al perfil del monitor, la teoría consultada en este estudio afirma que en otros países quienes laboran en los centros por alternancia son personas que tienen cierta especialidad en áreas técnicas y no sólo en docencia, en esta investigación se comprobó que, el mayor porcentaje son Maestros de Educación Primaria, también hay Bachilleres y Peritos haciendo docencia, pero un dato interesante es la mayoría de los docentes están siguiendo estudios universitarios.

En respuesta a la variable características de desarrollo generadas por la implementación del modelo, a pesar de las condiciones de funcionamiento de los NUFED, se evidencian diversas actividades dentro y fuera del aula que ya son un punto de partida hacia el desarrollo, aunque no de manera integral. Uno de los cursos técnicos que están implementando con mayor frecuencia es la agricultura a través de huertos escolares, familiares o de traspatio, observándose especies frutales como: limón, naranja y papaya, granos básicos como maíz, frijol y arroz; y verdura como berenjena, rábano, chile dulce, ayote, así como hierbas alimenticias.

Un dato interesante es que la comunidad ha empezado a consumir productos de las tiendas locales, y en el caso del maíz, frijol, algunas verduras y frutas están comprando lo que se produce en huertos familiares y escolares. Aunque los docentes consideran que la implementación de otro tipo de proyectos como bovino, aves o cerdos serían muy importantes para fortalecer el aprendizaje de los estudiantes y apoyo a las familias, no se realizan porque implican costos muy elevados, y por la falta de recursos económicos en las comunidades es difícil implementarlos, por muy pequeños que sean. 
No obstante, los docentes consideran que el modelo proporciona a los estudiantes las siguientes oportunidades: continuar estudios en el nivel medio, aprender un oficio, desarrollar actividades agrícolas, emprender pequeñas empresas para tener un mejor nivel de vida que los padres, contribuir con el desarrollo local. Aunque lo anterior es cuestionable cuando en este estudio se comprueba que los centros educativos están constituyendo un punto de partida para la continuidad de estudios de los jóvenes, y muy escasamente los están encaminando hacia una emprendeduría empresarial, u otro derivado de la formación por alternancia. Se están enfocando fundamentalmente a la enseñanza del área agrícola, pero de manera artesanal porque no tienen las condiciones necesarias para poder brindar una formación integral de acuerdo a todas las características que el modelo requiere.

Con la investigación se comprueba que los estudiantes egresados están sobresaliendo de manera personal, en las comunidades hay jóvenes que tienen una profesión como maestro, promotor de salud, perito contador, entre otras, lo cual es importante ya que con ese grado de preparación favorecen a sus familias y contribuyen con el desarrollo de la comunidad.

Los resultados de este estudio ayudan a confirmar los datos de algunas investigaciones que sostienen que el modelo por alternancia contribuye al desarrollo sostenible por medio del desarrollo humano (Chavén, 2007). Desde esa perspectiva, obtener información la ocupación de los jóvenes egresados de los NUFED fue interesante, hay evidencia estadística que la mayoría de egresados han podido continuar sus estudios y aunque muy pocos, algunos se han dedicado a la agricultura, lo anterior es interesante, sin embargo, no responde a la filosofía del modelo por alternancia, dado que la preparación académica es complemento de esa formación integral que el joven rural necesita para contribuir en el desarrollo de su medio; toda vez que se necesita empoderar a los jóvenes en la realización de proyectos productivos, pequeña empresa, y otros que sean un punto de partida hacia el desarrollo local.

Que existan una proporción de jóvenes dedicados al trabajo agrícola también es valioso para contribuir con las actividades productivas de la comunidad y con la seguridad alimentaria, quienes son agricultores, pero con formación académica que es precisamente lo que se esperaba del modelo según sus orígenes, y es dirigido a los jóvenes de escasos recursos de continuar estudios en el ciclo básico, principalmente a aquellos destinados a un contexto en donde solo se tienen acceso al nivel primario. El modelo propone un plan de formación para el medio de vida, que permite a los jóvenes involucrarse de manera activa en la solución de problemas de la comunidad, formación que les permite tener una perspectiva diferente a la de sus padres, que en su mayoría son de escasos recursos y al trabajo agrícola artesanal.

Finalmente, se resalta que a pesar de las limitantes que tienen los NUFED, se pudo verificar el esfuerzo que se realiza para el emprendimiento de actividades académicas relacionadas con la formación de las áreas técnicas, sin embargo, no son suficientes debido a que el objetivo de los NUFED no solo están orientados a la formación académica y el desarrollo de la persona, sino también, del medio de vida, por lo tanto, con los resultados de este estudio se comprueba que el modelo pedagógico por alternancia no está influyendo en el desarrollo local de una manera integral en las cinco comunidades rurales, y que al igual que los centros educativos tradicionales, están contribuyendo significativamente en la formación del recurso humano al brindar una opción de educación para los jóvenes del medio rural. 


\section{Conclusiones}

Con el estudio se confirma que el modelo pedagógico por alternancia no está contribuyendo con el desarrollo local de una forma integral, pero constituyen una alternativa para la formación del recurso humano lo cual también es un punto de partida para establecer las bases del desarrollo en el contexto rural.

La mayoría de directores consideran que el modelo pedagógico por alternancia contribuye de alguna manera con el desarrollo local, sin embargo, la metodología por sí sola no es suficiente debido a que se necesita la presencia de otros factores tales como: acompañamiento técnico pedagógico, el acceso a la tierra, a la tecnología, a los insumos y al apoyo crediticio por parte del Estado o de otras instituciones, para hacer posible el surgimiento de actividades que permita posicionar a los egresados de los NUFED como los nuevos actores del desarrollo en el contexto rural comunitario.

El estudio proporciona argumentos para hacer una evaluación del cumplimiento de las políticas educativas y la contribución del Ministerio de Educación, no obstante, aunque los NUFED necesitan mejorar sus condiciones de su funcionamiento de acuerdo con la filosofía del modelo, actualmente constituyen una oportunidad de formación para los jóvenes del área rural. 
Referencias bibliográficas

Aldana, C. (2010). Pedagogía para nuestro tiempo: Enfoque vivencial para estudiantes. Piedra Santa.

Arizmendy, A. y Escobedo, M. (2013). Legislación básica educativa. Centro de impresiones gráficas CIMGRA.

Bonilla, G. A. (2012). Desarrollo rural sustentable en Guatemala. Una perspectiva comunitaria. CUNSURORI.

Chavén, R. (2007). Alcances de la educación no formal, extraescolar o paralela en Guatemala.

Centro de Estudios para el Desarrollo Rural. (1998). Educación para el medio rural:

Figueroa de Teos, D. (2010). Calidad educativa y ampliación de la Educación Secundaria. Proyecto BIRF 7430-GU. Dirección General de Evaluación e Investigación Educativa, Ministerio de Educación.

García, R. (2002). La formación por alternancia en el medio rural: contexto e influencia de la FMR sobre el desarrollo local de Europa y los PVD modelo de planificación y aplicación al caso de Colombia. [Tesis doctoral, Universidad Politécnica de Madrid. Madrid, España]. http:// oa.upm.es/640/1/02200217.pdf

García, R. (2009). Educación, juventud y empleo. La alternancia, una alternativa para la educación y el desarrollo en América Latina. Serviprensa, AIMFR y UNESCO.

Lemus. L. (2007). Pedagogía: temas fundamentales. Piedra Santa.

Martínez, M. y Amador, L. (2010). Educación y desarrollo socioeconómico. Contextos educativos.

Menéndez, L. (2002). La educación en Guatemala 1954-2000. Enfoque histórico estadístico. Ediciones superación.

Ministerio de Educación de Guatemala. (2010). Currículum Nacional Base. Nivel Medio Ciclo Básico, Primer Grado. Articulación modalidad de alternancia, Núcleos Familiares Educativos para el Desarrollo NUFED. Dirección General de Educación Extraescolar.

Ministerio de Educación de Guatemala. (2013). Informe estudiantes de Estadísticas Educativas del ciclo básico del departamento de Petén, Guatemala.

Ortega, M. y Avendaño, O. (2012). Oferta de formación técnica para jóvenes: el Subsistema de Educación Extraescolar. ASIES.

Puig Calvó, P. (2006). Los centros de formación por alternancia: desarrollo de las personas y su medio. [Tesis doctoral, Universidad Internacional Catalunya, Barcelona, España]. https://www. tesisenred.net/bitstream/handle/10803/9345/Tesis.pdf?sequence=2\&isAllowed=y

Schwartz, N. B. (2010, 17 de julio). Algunas reflexiones acerca de la evolución de la sociedad petenera: cambio con o sin transformaciones. Conferencia presentada en el Centro Universitario de Petén, Flores, Guatemala.

Valenciano, J. y Gómez, A. (2001). Evolución de las teorías de desarrollo rural. La aplicación en España. Investigaciones sociales.

(C) Los autores. Este artículo es publicado por la Horizonte de la Ciencia de la Unidad de Posgrado de la Facultad de Educación de la Universidad Nacional del Centro del Perú. Este es un artículo de acceso abierto, distribuido bajo los términos de la Licencia Atribución-No Comercial 4.0 Internacional.(https://creativecommons.org/licenses/by-nc/4.o/), que permite el uso no comercial y distribución en cualquier medio, siempre que la obra original sea debidamente citada. 